\title{
Lymphogranuloma venereum: here to stay?
}

\author{
Helen Ward, ${ }^{1,2}$ Rob F Miller ${ }^{2}$
}

In 2003, this journal published a report from The Netherlands of an unusual presentation of lymphogranuloma venereum (LGV) in a man with HIV infection. ${ }^{1}$ That article was the first evidence of a significant re-emergence of the disease in gay men. Over the following 5 years, articles in this journal and others have documented cases and clusters of LGV from many countries in Europe and North America as well as from Australia and New Zealand. The cases have been in gay men, many of whom are coinfected with HIV and some with hepatitis C. The spectrum of clinical presentation has been wide, the majority having proctitis, rather fewer with classical urogenital syndrome and others being asymptomatic. ${ }^{2-4}$ Expanded diagnostic capacity, greater awareness among clinicians and patients, and new forms of surveillance have undoubtedly played a role in increasing detection of the disease.

\section{See linked articles, on p 165, p 171, p 173, p 176, p 180}

Despite 5 years of observation, many unanswered clinical and epidemiological questions remain, and five papers in this issue of Sexually Transmitted Infections address some of these. The first describes a unique case series from London of 13 men with LGV who had genital ulceration and inguinal syndrome. ${ }^{5}$ The details of these cases are of interest, since some were not "classical." Some of the patients had progressed to severe disease, and this

\footnotetext{
${ }^{1}$ Department of Infectious Disease Epidemiology, Imperial College London, London, UK; ${ }^{2}$ Sexually Transmitted Infections Editorial Office, BMA House, London, UK

Correspondence to: Dr Helen Ward, Department of Infectious Disease Epidemiology, Imperial College London, St Mary's Campus, Norfolk Place,

London W2 1PG, UK; h.ward@imperial.ac.uk
}

raises the possibility that cases of LGV are still being missed, particularly if they present with genital ulceration. The authors recommend swabbing genital ulcers in gay men for chlamydia, and considering LGV in people presenting with lymphadenopathy. The possible clinical spectrum is expanded further in a case report from France of a man with seroreactive arthritis apparently linked to LGV infection. ${ }^{6}$

A short report from Italy provides a picture of 13 cases diagnosed in Italy over a 2-year period. ${ }^{7}$ Early cases from 2006 reported sexual contact in other European countries, whereas more recently the infection appeared to be locally acquired.

A paper from the UK reports a multicentre case finding exercise among gay men attending genitourinary medicine clinics to establish whether there is a reservoir of undiagnosed infection that is being missed by current testing protocols. ${ }^{8}$ The paper reports a rectal LGV positivity of just less than 1\% in 6778 specimens, with almost all cases being symptomatic. In contrast, LGV was rare in the urethra with an estimated prevalence of less than $0.1 \%$. A linked study of chlamydia in gay men at a large centre in London found similar rates of LGV infection: $1.1 \%$ in the rectum and $0.03 \%$ in the urethra. ${ }^{9}$ They identified six asymptomatic cases of rectal LGV.

Many questions about LGV still remain, for example the exact mode and risks of transmission given the imbalance between genital and rectal infection, and the extent of the disease in the wider population. The high degree of coinfection with HIV is still a puzzle-possible explanations include biological synergy, density of sexual networks, differential case finding or a combination of factors.

For now, the recommendations regarding LGV remain as before: there needs to be a high degree of awareness and clinical suspicion, including of possible atypical presentations. People who have symptoms suggestive of LGV or who are contacts should be tested for chlamydia and positive isolates sent for typing. ${ }^{10}$ Treatment of suspected and confirmed cases and contacts should be with 3 weeks of doxycycline. A recent study of the persistence of different chlamydial serovars during the course of treatment supports the arguments that shorter courses of treatment are unlikely to be effective for LGV infection. ${ }^{11}$ In the light of the unanswered clinical and epidemiological questions, it looks likely that LGV is here to stay-at least for now.

Competing interests: HW and RM are editors of the journal, Sexually Transmitted Infections.

Accepted 29 April 2009

Sex Transm Infect 2009;85:157

doi:10.1136/sti.2009.037465

\section{REFERENCES}

1. Nieuwenhuis RF, Ossewaarde JM, van der Meijden $\mathrm{Wl}$, et al. Unusual presentation of early lymphogranuloma venereum in an HIV-1 infected patient: effective treatment with $1 \mathrm{~g}$ azithromycin Sex Transm Infect 2003;79:453-5.

2. White JA. Manifestations and management of lymphogranuloma venereum. Curr Opin Infect Dis 2009;22:57-66

3. Ward H, Martin I, Macdonald N, et al. Lymphogranuloma venereum in the United Kingdom. Clin Infect Dis 2007:44:26-32.

4. Van der Bij AK, Spaargaren J, Morre SA, et al. Diagnostic and clinical implications of anorectal lymphogranuloma venereumin men who have sex with men: a retrospective case-control study. Clin Infect Dis 2006:42:186-94.

5. Sethi G, Allason-Jones E, Richens J, et al. Lymphogranuloma venereum presenting as genital ulceration and inguinal syndrome in men who have sex with men in London, UK. Sex Transm Infect 2009;85:165-70.

6. El Karoui K, Méchaï F, Ribadeau-Dumas F, et al. Reactive arthritis associated with L2b lymphogranuloma venereum proctitis. Sex Transm Infect 2009;85:180-1.

7. Cusini M, Boneschi V, Arancio L, et al. Lymphogranuloma venereum: the Italian experience. Sex Transm Infect 2009;85:171-2.

8. Ward H, Alexander S, Carder C, et al. The prevalence of lymphogranuloma venereum infection in men who have sex with men: results of a multicentre case finding study. Sex Transm Infect 2009;85:173-5.

9. Annan NT, Sullivan AK, Nori A, et al. Rectal chlamydia - a reservoir of undiagnosed infection in men who have sex with men. Sex Transm Infect 2009;85:176-9.

10. Health Protection Agency. HPA surveillance and diagnostic protocols, 2009. http://www.hpa.org.uk/ webw/HPAweb\&PagegHPAwebAutoListName/Page/ $1191942171559 ? \mathrm{p}=1191942171559$ (accessed 29 Apr 2009).

11. de Vries HJ, Smelov V, Middelburg JG, et al. Delayed microbial cure of lymphogranuloma venereum proctitis with doxycycline treatment. Clin Infect Dis 2009;48:e53-6. 